\title{
Implementation of the Promoting Normal Birth Program in Iran - A Qualitative Study
}

\author{
Zohreh Sadat Hashemibonjar ${ }^{1}$, Rouhollah Zaboli², Nader Khalesi ${ }^{3}$, Ali Fakhr Movahedi ${ }^{4}$
}
${ }^{1}$ Department of Health Services Management, Semnan Branch, Islamic Azad University, Semnan, Iran. ${ }^{2}$ Department of Health Services Administration, Faculty of Health, Baqiyatallah University of Medical Sciences, Tehran, Iran.
${ }^{3}$ Department of Health Services Management, School of Medical Sciences, Islamic Azad University, Tehran, Iran.
${ }^{4}$ Department of Nursing, Nursing care research center, Semnan University of Medical Sciences, Semnan, Iran.

\section{ABSTRACT}

\section{BACKGROUND}

Normal birth is still highly valued, but the majority of pregnancies end with medical interventions or caesarean section. Rates of normal birth have been declining steadily over the past 20 years in Iran, despite the evidence of the benefits to mother and baby. According to the World Health Organization, the standard rate for caesarean is $10-15 \%$. In 2013, Iran Ministry of Health introduced the "Promoting Normal Birth Program". This study examines the views of experts about factors affecting the implementation of "Promoting Normal Birth Program".

\section{METHODS}

A qualitative study with framework analysis was used to determine the factors affecting implementation of the "Promoting Normal Birth Program" by examining views of 15 gynaecologists, anaesthesiologists, midwives and managers in 2018 at Zabol Medical Sciences University. Professionals participated with informed consent in this study. Data was collected from individual and group semi-structured interviews to reach saturation. MAXQDA 12 was used for analysis.

\section{RESULTS}

Seven categories were identified: (1) Leadership of Ministry, (2) Education, (3) Create Culture for a Normal Birth, (4) Optimization and Pleasure of Birth, (5) Normalization: The Essence of Birth and Midwifery, (6) Support, and (7) Empowerment.

\section{CONCLUSIONS}

These findings create a challenge for education, performance and health policies. In order to achieve program goals and improve women's health, the following actions are recommended- provide adequate infrastructure and financing to improve the birth environment, increase midwifery force, and promoting the culture of Normal birth, especially through the education for community, women, and caregivers, correcting rules and guidelines and detailing job descriptions and professional status.

\section{KEY WORDS}

Normal, Birth, Promoting, Qualitative

\begin{abstract}
Corresponding Author:
Rouhollah Zaboli,

Department of Health Services, Faculty of Health, Baqiyatallah University of Medical Sciences, Tehran, Iran.

E-mail: amirasjajs@gmail.com
\end{abstract}

DOI: 10.14260/jemds/2020/309

Financial or Other Competing Interests: None.

How to Cite This Article:

Hashemibonjar ZS, Zoboli R, Khalesi N, et al. Implementation of the promoting normal birth program in Iran- a qualitative study. J. Evolution Med. Dent. Sci. 2020;9(17):1416-1422, DOI: 10.14260/jemds/2020/309

Submission 02-10-2019,

Peer Review 15-12-2019,

Acceptance 21-12-2019,

Published 20-03-2020.

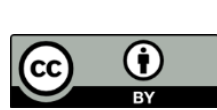




\section{BACKGROUND}

Providing high-quality health care has always been and will be the most important concern of governments. Because, in the contemporary world, health is the second most important need of nations after security.[1] That is why, over the past two decades, there has been a strong international trend towards health reform with particular attention to the health of women in many countries.[2] In Iran, the Health System Reform Plan has been implemented over five years. ${ }^{[3]}$ Rates of normal birth have been declining steadily over the past decades. In many countries, policy-makers are working to promote normal birth.[4] Iran Ministry of Health has implemented the "Promoting Normal Birth Program" in the Health System Reform Plan to increase normal childbirths. ${ }^{[3,}$ 5]

Caesarean is one of the most common surgeries in the world, which is increasing in developed and developing countries. Although this surgery is essential in emergency cases, many women give birth by Caesarean with no comprehensive medical reasons.[6] . According to the World Health Organization, the standard rate for caesarean is 1015\%.[6-8] The World Health Organization, reported, the rate of Caesarean $41.9 \%$ at 2010 , and $88 \%$ in private hospitals and $47 \%$ in government hospitals at 2013 in Iran.[7, 9] The "Promoting Normal Birth Program" was implemented at May 2014, to decrease Caesarean in Iran.[6] In this program, various actions has considered for promotion of Normal Birth, such as optimization of the maternity setting environment, the implementation of Prepared birth classes, the provision of incentive payments for gynaecologists, midwives, and anaesthesiologists, charge free Normal Birth in government hospitals. $[10,11]$

In this program, pregnancy and birth are considered as a pleasant experience in a natural process which is provided by a team of medical experts during pregnancy and birth.[12,13] In this program" to increase the willingness of gynaecologists for Normal Births, preferential tariffs for Normal Birth versus Caesarean has been considered and normal birth tariffs increased and were closer to caesarean tariffs. Therefore, the most appropriate financial strategy is used to control the increasing trend to caesarean. [14] Research on new health programs is an important factor in improving the performance of it,[15] and will provide an overview of the health system and guidance for health policies.[16] Also, it provides practical interventions to improve the program and its components and creating a public accountability culture. In several countries such health reforms have taken place, research has played a major role in guiding reform and monitoring its progress. $[17,18]$

In this paper, we explored views of gynaecologists, midwives, anaesthesiologists and managers about the factors affecting the implementation of "Promoting Normal Birth Program".

\section{METHODS}

A qualitative study aimed to determine the views of gynaecologists, midwives, anaesthesiologists and managers about the factors affecting implementation of "Promoting Normal Birth Program" at Zabol Medical Sciences University.
The study approach is analytical framework, because researchers wanted to manage and organize data to create a new structure to summarize a national program. We intended to provide a systematic model for managing and mapping the data and presenting a model. Framework analysis was considered to be a better choice than thematic analysis, because of that fitted the aims of our study[19]. The sample population included 15 gynaecologists, midwives, anaesthesiologists and managers with their consent, about explaining change factors in the current program. The purposeful sampling method was used. All of participants were aware of the various stages of program. As this program was only implemented in government sector, no participants were sought from the private sector.

Participants with at least 10 years of experience were included in the study. Participants reluctant to continue in the study were excluded. Data was collected by semi-structured interviews of individuals and groups, which were conducted at a time and place that was convenient to each participant. Since contributors were selected from different categories, focus group interviews were conducted to express their views. Five to six people participated in these sessions. Each interview lasted between $45-85$ minutes and proceeded to saturation. The interviews and focus groups were audio recorded, transcribed verbatim and analysed with MAXQDA 12.

Data analysis was performed, along with data collection, on the basis of framework analysis. In this method, codes and classes were extracted directly and inductively from raw data. Immediately after each interview done, the text was typed word by word. To identify semantic units, each interview was studied and reviewed several times. The results were shared with the participants and their feedback was requested. Subsequently, semantic units were reviewed several times and classified according to conceptual similarity. Themes and sub-themes were compared together and extracted. Six faculty members (check peer) validated the data. In this study, one researcher collected and analysed all the data, with regular discussion of the finding with a mentor. Ongoing analysis and continuing engagement of the researcher with themes and subthemes was done.

\section{Ethical Statement}

We received ethical approval from the Ethics Committee of Semnan Branch, Islamic Azad University for this study (approval number: IR.IAU.SEMNAN.REC.1396.9). Also, The Local Ethical Committee approval was obtained from ZBMU. All participants received written and oral information about the study. Written consent was obtained from all participants. Participants were informed that participation was voluntary and ensured confidentiality.

\section{RESULTS}

This study explains the factors affecting the implementation of "Promoting Normal Birth Program". Fifteen participate in this study. The mean age of participants was 46.6 and the average work experience was 20.6 years. Their demographic characteristics are given in the table 1. 


\begin{tabular}{|c|c|c|c|c|}
\hline No. & Job Title & Job Category & $\begin{array}{c}\text { Work } \\
\text { Experience } \\
\text { (Years) }\end{array}$ & $\begin{array}{c}\text { Age } \\
\text { (Yrs.) }\end{array}$ \\
\hline 9 & Midwife & $\begin{array}{c}\text { Health section: 2, Treatmentsection: } 4 \\
\text { Educationdepartment (faculty): } 3\end{array}$ & $21.7(13-31)$ & $(53-33) 45.4$ \\
\hline 3 & Gynaecologist & Associate Professor: 2, Gynaecologist 1 & $19.3(12-24)$ & $(45-53) 48.3$ \\
\hline 2 & Manager & Associate Professor & & \\
\hline 1 & Anaesthesiologist & Associate Professor & & \\
\hline \multicolumn{5}{|c|}{ Table 1. Demographic Characteristics of Participants } \\
\hline \multicolumn{5}{|c|}{} \\
\hline
\end{tabular}

In total, 1556 codes explored. Seven categories were identified- (1) Leadership of ministry, (2) Education, (3) Create Culture for Normal Birth, (4) optimization and pleasure of birth, (5) Normalization: the essence of birth and midwifery, (6) Support, and (7) Empowerment. Each of these Categories has Themes and Several Sub-Themes are described in Table 2

\begin{tabular}{|c|c|c|}
\hline Categories & Themes & $\begin{array}{l}\text { Subthemes } \\
\end{array}$ \\
\hline \multirow{5}{*}{$\begin{array}{c}\text { Leadership of } \\
\text { the ministry }\end{array}$} & Policy making & $\begin{array}{c}\text { Description of transparent tasks, legislation and clear } \\
\text { protocols, assignment health services to private section } \\
\text { and supervise }\end{array}$ \\
\hline & Financing & $\begin{array}{c}\text { Public insurance, free normal childbirth, staff financing } \\
\text { \& on time incentive payment }\end{array}$ \\
\hline & \begin{tabular}{|c|} 
Necessary infrastructure \\
for the "Promoting Normal \\
Birth Program" \\
\end{tabular} & $\begin{array}{l}\text { Improvement of labour blocks, } \\
\text { Electronic Health Record Systems (SEPAS,SIB) }\end{array}$ \\
\hline & \begin{tabular}{|l|} 
Employee development \\
\end{tabular} & Reduce staff burnout, legal protection of staff \\
\hline & Supervision \& evaluation & $\begin{array}{c}\text { Monitoring and follow up on caesarean, incorrect } \\
\text { statistics of caesarean }\end{array}$ \\
\hline \multirow{4}{*}{ Education } & MotherEducation & Birth preparation classes \\
\hline & Family Education & $\begin{array}{l}\text { Spouse education } \\
\end{array}$ \\
\hline & Care providers Education & Continuing education of caregivers, Student education \\
\hline & Community Education & Media, TV \\
\hline \multirow{4}{*}{ Optimization } & \begin{tabular}{c|} 
Providing mental, \\
emotional, physical and \\
structural for mother and \\
her family
\end{tabular} & $\begin{array}{l}\text { Psychological and emotional well-being, Improvement } \\
\text { of maternity settings (adequate rooms \& beds, privacy, } \\
\text { pain management), Attendant (Midwife, Doula, one of } \\
\text { the family) }\end{array}$ \\
\hline & Optimization forStaff & Intimate atmosphere, Lack of task-shifting \\
\hline & Labour pain management & $\begin{array}{c}\text { Non-pharmacological methods for pain reduction } \\
\text { pharmacological methods for pain reduction, } \\
\text { cooperation of anaesthesiologists }\end{array}$ \\
\hline & Continuity of care & Caring continuity, care provider continuity \\
\hline \multirow{3}{*}{$\begin{array}{c}\text { Create Culture } \\
\text { for the Normal } \\
\text { Birth }\end{array}$} & $\begin{array}{c}\text { Creating a belief in normal } \\
\text { nature ofbirth }\end{array}$ & $\begin{array}{c}\text { Stop normal birth medicalization, medical paternalism, } \\
\text { reduce fertility and golden baby }\end{array}$ \\
\hline & $\begin{array}{c}\begin{array}{c}\text { Education and advertising } \\
\text { for Normal Birth }\end{array} \\
\end{array}$ & $\begin{array}{l}\text { Caesarean sections: a sign of modernity, Caesarean } \\
\text { sections in celebrities, doctors, and midwives }\end{array}$ \\
\hline & \begin{tabular}{|l|} 
Community Education \\
\end{tabular} & Primgravida education, teenager's education \\
\hline \multirow{2}{*}{\begin{tabular}{|c|} 
Normalization: \\
the essence of \\
birth and \\
midwifery \\
\end{tabular}} & $\begin{array}{c}\text { Correct understanding of } \\
\text { Normal Birth }\end{array}$ & Meaning of normal birth, non-intervention \\
\hline & $\begin{array}{c}\text { Midwife as trustee of } \\
\text { maternity care }\end{array}$ & $\begin{array}{l}\text { Midwife features: skill, autonomy, patience, trusted } \\
\text { relationship, task-shifting }\end{array}$ \\
\hline \multirow{3}{*}{ Supporting } & $\begin{array}{l}\text { Mother \& Family } \\
\text { supporting }\end{array}$ & $\begin{array}{l}\text { Information support (education, preparation classes for } \\
\text { birth), emotional support (reducing stress, attendant or } \\
\text { doula, dignity), physical support: safe and pleasant } \\
\text { environment, pain relief }\end{array}$ \\
\hline & Care providers support & $\begin{array}{l}\text { Burnout, task-shifting, fear of litigation 4. Continuous } \\
\text { education }\end{array}$ \\
\hline & Community supporting & $\begin{array}{c}\text { Community financial support (public insurance), } \\
\text { insurance of midwifery services }\end{array}$ \\
\hline \multirow{3}{*}{$\begin{array}{l}\text { Empowerment } \\
\text { of all: }\end{array}$} & Promoting awareness & $\begin{array}{l}\text { Mother and family education (preparation classes for } \\
\text { birth) }\end{array}$ \\
\hline & $\begin{array}{l}\text { Reduce fear \& } \\
\text { stress }\end{array}$ & $\begin{array}{c}\text { Fear of labour pain, fear of loneliness, fear of the } \\
\text { maternity environment, fear of interventions }\end{array}$ \\
\hline & Choice of birth method & $\begin{array}{l}\text { Fear of birth, education, effect of family, caregiver, } \\
\text { community, impact of experience, demographic factors } \\
\text { (age, education, income, etc.), culture and beliefs }\end{array}$ \\
\hline
\end{tabular}

Policy Making- Contributors commented about the ministry's role in providing infrastructure, defining job descriptions.

P9- The plan is fair. There is no doubt that normal birth is better than caesarean, but the infrastructure is not satisfying The boundaries of tasks are not clear. Autonomy of a midwife is undermined. They believed, the duties of a midwife and gynaecologist should be clearly explained and there should be no task-shifting between them.
Legislation and Clear Protocols- Iran ministry of health has published national protocols for maternity care. Despite of these guidelines, participants shared their views on the need to establish clear and practical protocols based on program policies. They said the program rules, the patient's rights, and the incentive payment rules are not respected.

Financing- The majority of participants believed that funding of the program started well, but over time; the government faced a budget shortage. Inability to pay timely caused a delay in staff payments, equipping hospitals, and building birth units. All of the participants welcomed the national health insurance in the program. Also, two participants stated that timely payment would encourage gynaecologists to provide high-quality care. They mentioned free childbirth in government hospitals could promote safe normal birth, especially for many rural and low-income women.

P4- "One of the most important benefits of this program is a reduction in maternity payments. Because many mothers really cannot pay for normal birth, and especially, they can't give birth in more equipped and better hospitals, but the majority of mothers are encouraged to deliver in hospitals now which are equipped, because it's free.

Free Normal Childbirth- Some participants disagreed with free childbirth and believed that it should be just for lowincome groups:

P5- "In my opinion, the free normal childbirth is wrong. Free services are meaningless. Maybe this is for increasing birth; while this is also wrong."

Staff Payments- They believed incentive payments would encourage caregivers to facilitate the program:

P6- "Now, the most important issue is financing of the program. Consider a gynaecologist works in Hirmand, only because of financial benefits. Otherwise, if we don't pay well, a gynaecologist will never work at a small town that its distance to the border is only 700 meters,

Provide Necessary Infrastructure for "Promoting Normal Birth Program"- The participants have commented on the lack of program infrastructure, poor availability of birth preparation classes, shortage of staff, beds, and hospital spaces, the lack of facilities for normal birth and mother attendant in maternity wards.

P7- "We have a lot of problems because of the lack of infrastructure. When the ministry had been started the program, there was no infrastructure, no money, and... in my opinion, the program has failed."

Electronic Health Record System- The majority of professionals mentioned that although electronic health records such as SIB and SEPAS have improved quality and efficiency of maternity care, it is time-consuming and because of the staff shortage, it is difficult for them to deal with it. 
P2- "Since 2015, we have had electronic records. We have a system called SIB where the services are recorded. Of course, there are problems with the infrastructure, sometimes, internet is disconnected or interrupted." The number of records registered in SIB system is a criterion for evaluating midwives' practice. The midwives stated that due to a large number of clients, they do not have enough time to take care of mothers and spend too much time registering people on the system.

P10- "All midwives just record the data in the SIB system. Their work is just ticking, no thinking, like a robot. ... The quality of care does not matter, only the number of cases and quantity are important."

Legal Protection of Staff- Legal protection for carers reduces the fear of legislation and facilitates the normal birth. They stated midwives alone take birth of babies, while gynaecologists are not present in labour and birth rooms. But midwives have to register all of the deliveries by the name of gynaecologists beside their names; it removes their incentives and self-confidence.

Supervision- Five participants believed that program supervision was weak. A manager believed that the ministry's expectations should be based on facilities.

P11- "When you talk about normal birth, look at the environment. Look at what's happening in this program. Are your expectations consistent with the facilities and conditions hospitals?". But a participant said about good ministry supervising on caesarean sections:

P7- "The ministry has a lot of oversight, on caesarean. My caesarean sections are almost $20-19 \%$. So, no one has warned me so far. But authorities warned my colleagues that they did too many caesarean sections. The participants stressed the importance of mothers and family education. All of them emphasized the importance of Birth preparation classes and considered them as the strengths of the program. They stressed on continuing education for midwives and gynaecologists to promote normal birth.

Optimization of Normal Birth- The birth environment should be physical, emotionally, and socially attractive for mothers and care providers. They said the maternity hospital constraints affect the normal process of birth, for example inadequate and inappropriate rooms and beds, burnout, and the multiplicity of midwife duties increase the medical intervention through speeding up labours. The crowded maternity ward was a barrier for normal birth. Especially in educational hospitals, the presence of medical and midwifery students was considered a reason for mother's fear and lack of progress in birth.

P13- "The mothers complain of frequent and continuing examinations, they are tired of the staff's bad behaviour, the repeated history taking by students in various shifts, and all of these factors make patients to complain."
Create Culture for the Normal Birth- Community culture and beliefs can affect normal birth; therefore, it is needed to create a belief in the normal nature of birth. Nowadays, many women prefer caesarean. they think caesarean is fashion or it is a sign of modernity because women with higher incomes and socioeconomic classes choose caesarean.

P1- "We should make a normal birth culture. It takes time. Cultural change is difficult, especially with this generation who sees modernity in caesarean." The midwife's belief in the naturalness of birth can be transferred to mothers.

P6- "Midwives more likely help mothers to give birth normally, while women who go to gynaecologists more likely to deliver by caesarean." Most of them believed technological overcoming and interventions of gynaecologists caused women think pregnancy and birth should be treated as a disease.

P15:- "Now women are thinking that pregnancy and birth are dangerous, and we have to do something for them. They come to my office and say: Do we need any medication?" Another issue is extreme medical paternalism, and underestimate of midwives, which is applied by the Ministry of Health.

P9- "Medicine overcoming exists in our health system: medical paternalism. Midwives not allowed working independently."

Normalization: The Essence of Birth and Midwifery- The participants believed naturalness was neglected in the true sense of the word. Normal birth refers to birth without intervention. Moreover, when the care provider does not intend to intervene, maternity unit conditions such as limited birth beds, a lot of patients, and a shortage of midwives do not allow waiting for the normal birth. They stressed the midwife role as a trustee of maternity care:

P13- "Midwives have natural and divine work because they are" Guardian of Creation".

Supporting- The participants discussed the supporting of the mother, family, caregivers, and social support in three dimensions of informational (education and birth preparation classes), emotional (good communication, confidence between mothers, her family, and caregivers, an attendant along with mother, reduced labour pain), and physical (pleasant environment of birth) counselling. They said carers should be supported by stopping burnout with sufficient staff employing, preventing multiple duties, ensuring the job security, no fear of litigation, and informational support provided by the continuing education. In social supporting issues, funding for maternity care (free birth) and community insurance was emphasized. The participants also considered if midwifery services are covered by insurance, it will support midwives and promote normal birth.

Empowerment- Contributors stated that the program has empowered women to choose their birth method through 
education and participation in Birth preparation classes. Furthermore, reducing stress and fear of mother. This fear can be eliminated by empowering mothers using labour pain relief, pleasant environment, accompanying with mother and avoiding unwanted interventions.

\section{DISCUSSION}

Figure 1 shows the conceptual model of the factors influencing the program for the promotion of normal birth from the views of the participants and the relationship between them:

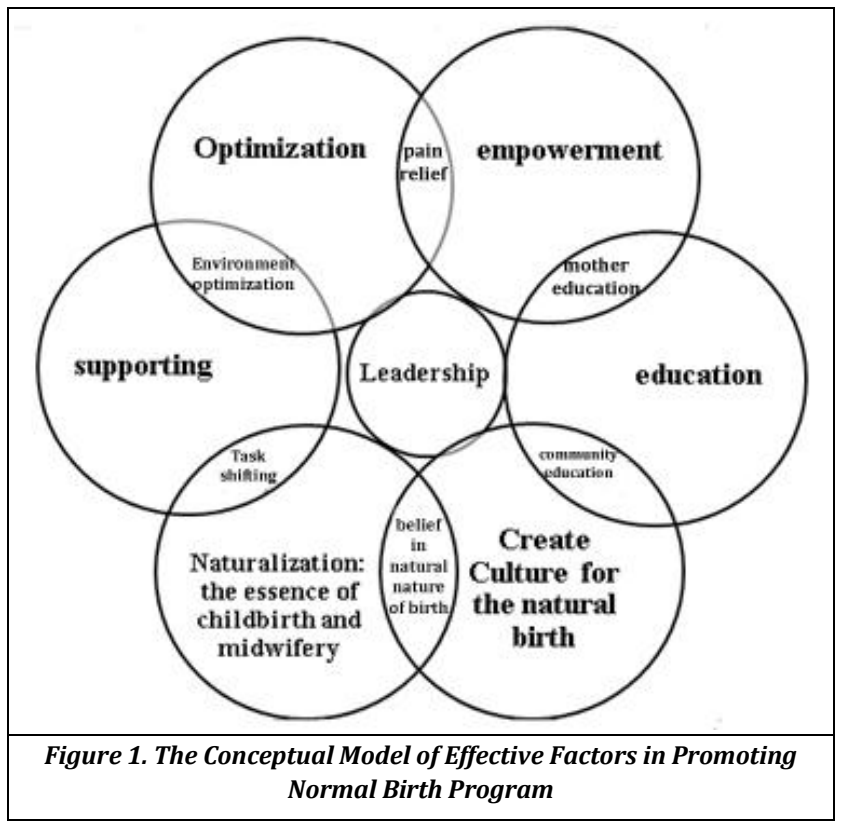

As you see, these themes are joined together like chain loops and overlap in some categories, While Leadership of the Health Ministry is centred. According to World Health Organization, a good health system works to coordinate with efficient and motivated staff in an appropriately maintained infrastructure, with trusted medicines and technology, with adequate funding and strong health plans and evidencebased policies are supported.[18] The leadership of the health system along with the provision of resources, financing, and services is one of the four core functions of a health system and has a direct impact on other functions.[20] The major health problems of developing countries is that they make regulations and standards without regard to clients and caregivers as stakeholders in policy-making and they do not care other components of leadership such as knowledge production, implementation, and monitoring of policies, Particularly ignore some issues, such as under the table payments or controlling the behaviour of caregivers. $[19,20]$ The results of this study also indicate that the ministry's leadership should pay more attention to providing funding, proper policy and appropriate legislation for the effective implementation of this program.

The participants reported that electronic documentation is a time-consuming process may affect care quality and critical thinking skills of midwives. A systematic review of electronic nursing documentation suggests that future research studies should be done to examine the impact of electronic documentation on the quality of care.[13] Although midwifery is an independent profession in Iran,[14] midwives cannot be autonomous due to the increasing medical interventions by gynaecologists, medical dominance and rules weaknesses, and they should work under the supervision of the gynaecologist. A midwife is not given the authority to sign her name as the main responsible for normal birth and it should be recorded in the name of gynaecologist and midwife in the patient's case.[17]

Mothers' education is one of the important points in reducing the rate of caesarean. Birth preparation classes are considered in the program. Mehdizadeh et al. showed that the rate of caesarean in a group participated in Birth preparation classes was 15\% lower than the control group.[21] The results of the studies indicate that Birth preparation classes are an unrivalled opportunity to help women learn how to live healthy.[13] The burden of midwife's responsibilities, the lack of time, workload and multiple duties, shortage of staff, do not let them perform birth preparation classes.This is similar to findings of others.[15,16] Optimization of the environment is considered in the program not implemented due to failure to provide infrastructures. In order to support normal birth, the physical, emotional, and social environment of birth should be attractive for women and care providers. The emotional environment involves the thoughts, feelings, and responses of care providers and women. These results are consistent with the study by Hammond et al. (2013).[22] We found that the task-shifting between carers affects their autonomy, so health policy-makers should clearly outline the responsibilities of carers to avoid interfering with their duties.

Colvin et al. (2013) concluded that task shifting requires ongoing supervision and support as well as careful integration into clinical protocols and the broad shifting with care. Also task shifting should be considered when rewriting the roles and responsibilities of the midwife, to anticipate the inter-professional conflicts, responsibility ambiguities, and social hierarchies between care providers.[25] In our study, Normal Birth "was interpreted as a physiological approach with the least dependence on technology and a greater dependence on emotional support, which is consistent with the World Health Organization and International Confederation of Midwives definition.[26-27]

Evidence suggests that intervention in normal physiological process of labour and birth increases the risk of maternal and infant complications. Therefore, the promotion, protection, and maintenance of normal labour is a challenge to respect the basic physiology of pregnancy.[28] The midwife, as a trustee of maternity care, knows and can manage normal labour and birth. She is specialized in normal labour and birth and believes birth runs naturally. These results are also consistent with studies done by Davis (2010) and Dahlberg $\left(2016 .{ }^{29,30]}\right.$ Although the program stipulates that facilities for normal birth by private midwives should be provided at government hospitals, $[10,11]$ this has not been granted to private midwives.

Rigg et al. stated that lack of supporting private midwives, leads to unregulated birth workers to undertake many practices that should be done by registered midwives.[31] They agreed that the community's culture influenced the belief in the natural process of birth. Latif Nejad et al, also Bagheri et al., Showed that society's culture affects the choice 
of birth in women. ${ }^{[32,33,34]}$ Also, the medicalization of birth and excessive reliance on risk in normal birth lead to unnecessary interventions that do not benefit the mother and foetus. Our study is consistent with the results of others. ${ }^{[32,33]}$ Carolan and Hodont (2007) indicated that the medicalization of birth is contradictory to the normal nature of birth which leads to a complex network of monitoring and caring.[36]

Increasing normal birth tariffs in the program have led to an increase in the normal birth rate. Although this is a good result of the program, birthing with gynaecologists lead to medicine dominance and unnecessary interventions in birth.[34] Participants pointed to the brilliant role of gynaecologists in the maternity ward, indicating extreme medical paternalism imposed by the Ministry of Health and, ultimately, the organization. The results showed that some factors related to the community culture could have a facilitating role in Caesarean sections. Apparently, in our society, the caesarean has become a fashion. When social habits are gradually established, it's difficult to change them. People think that caesarean is more likely to help them to have a healthy baby. A desire for caesarean in educating women and doctors promotes this belief this way is safer and better than a normal birth. This finding is consistent with Bagheri et al. (2012) and Latif Nejad et al.

(2015) studies.[32,33]

Sometimes women are also seeking caesarean because they are stigmatized as a low-income class that accompanying with Orfani (2012) in India..[3] According to the participants, in order to make culture and the belief in the normal nature of birth, the knowledge and attitude of women, families, carers and other people involved in women's health should be promoted by education. Education was seen as a critical component that empowering women to make informed choices about their birth methods. Women's empowerment is not just about education. Many other factors, such as culture, role of women in society, women's self-esteem and awareness of existing facilities to help her in birth, have also an important role in empowering women.

\section{Limitations}

This is a qualitative research, and qualitative designs focus on meanings and understanding. The number of small samples involved can limit the generalization of the findings. The other limitation of the study was that the study was conducted only in a university and only care providers participated in it and mothers did not attend. Moreover, the actual birth environment was not observed. The strength of this study was the presence of professionals from all fields of midwives, and other involved staff participated in this study.

\section{CONCLUSIONS}

The implementation of this program is associated with challenges such as cultural and financial affairs, but in general, it has been able to overcome the unceasing growth of Caesarean in our country. As the participants mentioned, the existing policy implementation is poor, partly due to limited funding. There is an opportunity for policymakers to take a leading role to improve utilization of this program from the highest level in both public and private hospitals. This can be achieved through making and disseminating appropriate policies, improving staffing supervision and creating, enabling and optimizing the environment for normal birth. [39] Also, evaluating the efficacy of normal birth training courses, assessing the satisfaction of mothers and their families from birth wards and services may be helpful. Promoting the culture of normal birth should also be done through conferences, seminars, television advertising, and adolescent education. Joining of private hospitals, social security insurance, and care providers especially gynaecologists, to the program, should be encouraged. Paying attention to education-topics about physiological birth can also improve program achievementsI $n$ order to improve normal birth, the natural process of birth should be emphasized, and the normal birth culture should be promoted. In addition, the midwife, as a trustee of normal birth, should be legally supported by describing clearly and precisely their duties should prevent task-shifting between them and obstetricians.

\section{REFERENCES}

[1] Esmailzadeh, H., et al., Iran Health System Reform Plan Methodology. Iran J Public Health, 2013. 42(Supple1): p. 13-17.

[2] Heidarian, N. and S. Vahdat, The impact of implantation of Health Care reform plan in patients pay out of pocket in selected Public hospitals in Isfahan. Journal of Medical Council of Iran, 2015. 33(3): p. 194-187.

[3] Gharibi, F., et al., A Survey of Health System Reform Circumstances from the Experiences of Managers and Nurses of Tabriz Taleqani Hospital. Depiction of Health, 2016. 6(1): p. 1-10.

[4] Farahani, L.A. and M.J.A. Shavazi, Caesarean Section Change Trends in Iran and Some Demographic Factors Associated with them in the Past Three Decades. Journal of Fasa University of Medical Sciences, 2012. 2(3): p. 127-34.

[5] Piroozi, B., et al., Evaluating the effect of health sector evolution plan on caesarean rate and the average costs paid by mothers: A case study in Kurdistan province between 2013-2015. Hayat, 2016. 22(3): p. 245-254.

[6] Monk, A., et al., Australian primary maternity units: Past, present and future. Women and Birth 2013. 26(213218).

[7] World Health Organization, W., warning about the development of caesarean section, WORLD HEALTH ORGANIZATION statement on caesarean section rates. 2015: Geneva, Switzerland.

[8] Ghahfarokhi, Z.S. and F. Khalajabadi-Farahani, Intention for Caesarean Section Versus Vaginal Delivery Among Pregnant Women in Isfahan: Correlates and Determinants. J Reprod Infertil. 2016 Oct-Dec; 17(4): 230-239.

[9] Dehghan, A., et al., Performance of Health Care System Reform Plan From the Perspective of University Hospitals Executives in Yazd Province in 2015. Manage Strat Health Syst., 2016. 1(1): p. 43-49. 
[10] Education, M.o.H.a.M., The most important actions annual report of the Ministry of Health and Medical Education of Iran in the eleventh goverment. 2014.

[11] Razavi, S.E., Health system reform plan in Iran: Approaching Universal Health Coverage. Hakim Health Sys Res, 2016. 18(4): p. 329-335.

[12] g.f.t.P.N.B.P. Iran Ministry of Health and Medical Education - Deputy Director of Treatment. A summary of guidelines for the Health System Reform Plan, Editor. 2013.

[13] Education, M.o.H.a.M., The most important actions reported three-year, the Ministry of Health and Medical Education in the eleventh goverment. 2016.

[14] Babaei, F., et al., Study of the Promotion of Normal Delivery Program in Government Hospitals in Line with the Health Transformation Plan and Its Achievements. Hakim Health Sys Res. 2017; 20 (1):44-53.

[15] Sang, S., Z. Wang, and C. Yu, Evaluation of Health Care System Reform in Hubei Province, China. Int. J. Environ. Res. Public Health, 2014. 11(2): p. 2262-2277.

[16] Fleuren, M., K. Wiefferink, and T. Paulussen, Determinants of innovation within health care organizations Literature review and Delphi study. Int J Qual Health Care 2004. 16(2): p. 107-123.

[17] WORLD HEALTH ORGANIZATION, Strategy on Health Policy and Systems Research: Changing Mindsets.: World Health Organization;. 2013, Geneva.

[18] Rao, K.D., R. Arora, and A. Ghaffar, Health systems research in the time of health system reform in India: a review. Health Res Policy Syst, 2014. 12(37).

[19] Sandelowski, M., S. Docherty, and C. Emden, Focus on qualitative methods.Qualitative metasynthesis: issues and techniques. Res Nurs Health.1997;20(4):365-71.

[20] WORLD HEALTH ORGANIZATION. Health systems. 2018; Available from: https://www.World Health Organization.int/healthsystems/news/update/en/.

[21] Health Systems Profile- Islamic Republic of Iran., in World Health Organization. 2006.

[22] Biglar, M., P. Bastani, and S.V. khah, The Challenges of Stewardship In Medical Education System: A Qualitative Approach. payavard Salamat, 2013. 7(4).

[23] Mehdizadeh, A., et al., Evaluation of the effectiveness of antenatal preparation for childbirth courses on the health of the mother and the newborn. Journal of Iran University of Medical Sciences, 2003. 10(35): p. 455-62.

[24] Hammond, A., et al., Space, place and the midwife: Exploring the relationship between the birth environment, neurobiology and midwifery practice Women and Birth 26 (2013) 277-281.

[25] Colvin, C.J., et al., A systematic review of qualitative evidence on barriers and facilitators to the implementation of task-shifting in midwifery services. Midwifery, 2013. 29: p. 1211-1221.
[26] WORLD HEALTH ORGANIZATION, Care in normal birth: A practical guide, World Health Organization,Reproductive Health and Research. 1999: Geneva. p. 4.

[27] Romano, A.M. and J.A. Lothian, Promoting, Protecting, and Supporting Normal Birth: A Look at the Evidence. JOGNN 2008. 37: p. 94-105.

[28] Kennedy, H.P. and T.P. Shannon, Keeping birth normal. Clinical Research, 2004. 3: p. 554-560.

[29] Davis, J.A.P., Midwives and Normalcy in Childbirth: A Phenomenologic Concept Development Stud,. J Midwifery Womens Health, 2010. 55:206-215.

[30] Dahlberg, U., et al., How can midwives promote a normal birth and a positive birth experience? The experience of first-time Norwegian mothers. Sexual Reproductive Healthcare 2016. 7(2-7).

[31] Rigg, E.C., et al., The role, practice and training of unregulated birth workers in Australia: A mixed methods study. Women Birth. 2019 Feb;32(1):e77-e87.

[32] Bagheri, A., N.M.-. Alavi, and F. Abbaszade, Effective factors for choosing the delivery method among the pregnant women in Kashan Feyz, 2012. 16(2): p. 146153.

[33] Roudsari, R.L., M. Zakerihamidi, and E.M. Khoei, SocioCultural Beliefs, Values and Traditions Regarding Women's Preferred Mode of Birth in the North of Iran. Int J Community Based Nurs Midwifery, 2015 Jul. 3(3): p. 165-176.

[34] Roudsari, R.L., et al., Comparing the Cultural Beliefs related to Mode of Delivery among Pregnant Women and Women with Childbirth Experiences as vaginal delivery or caesarean section (Tonekabon, 2014. J Mazandaran Univ Med Sci. 2015; 24 (120):54-68.

[35] Downe, S., K. Finlayson, and A. Fleming, Creating a collabourative culture in maternity care. J Midwifery Womens Health, 2010. 55(3): p. 250-4.

[36] Carolan, M. and E. Hodnett, With woman" philosophy: Examining the evidence, answering the questions. Nursing Inquiry 14(2), 140-152. 2007.

[37] Reiger, K., A neoliberal quickstep: contradictions in Australian maternity. policy. Health Sociology Review, 2006. 15: p. 300-340.

[38] Orfali, K., Beyond numbers: the multiple cultural meanings of rising caesarean rates worldwide. Am J Bioeth, 2012. 12((7)): p. 54-6.

[39] Owen, O.D., Access to health care in developing countries: breaking down demand side barriers Cad. Saúde Pública, Rio de Janeiro, 23(12):2820-2834, dez 2007. 